respectively. One-half recurrences had occurred within 6 months, and almost $90 \%$ within 2 years. Risk factors for seizure recurrences included a remote symptomatic etiology, abnormal EEG, nocturnal seizures, prior febrile seizures, and Todd's paresis. Children with the most favorable prognosis $(21 \%$ recurrence risk after 5 years) had a cryptogenic first seizure while awake and a normal EEG. (Shinnar S, Berg AT, Moshe SL et al. The risk of seizure recurrence after a first unprovoked afebrile seizure in childhood: an extended follow-up. Pediatrics Aug 1996;98:216-225). (Reprints: Shlomo Shinnar MD, Epilepsy Management Center, Montefiore Medical Center, 111 E 210th St, Bronx, NY 10467).

COMMENT. This study is important because of the large number of patients included and the long duration of follow-up. In addition to acquired symptomatic etiology and EEG dysrhythmia, factors frequently associated with an increased risk of seizure recurrence in other studies, sleep related seizures, prior febrile seizures, and Todd's paresis were demonstrated as risk factors. Cryptogenic seizures while awake and a normal EEG were predictive of a good prognosis and a low rate of recurrence.

In a further report, these authors correctly point out that the control of seizures by AEDs and a favorable long-term remission is only part of the goal of therapy. Heightened self-esteem, improved school achievement, and social acceptance by peers are other important considerations. Although the prognosis of epilepsy is determined largely by the underlying cause and associated neurologic abnormalities, the control of seizures by AEDs improves the quality of life and is justification for rational therapy. (Shinnar S, Berg AT. Does antiepileptic drug therapy prevent the development of "chronic" epilepsy? Epilepsia Aug 1996;37:701-708).

\title{
SUDDEN UNEXPLAINED DEATH AND EPILEPSY
}

The incidence of sudden unexplained death (SUD) among patients less than 50 years old with refractory epilepsy was determined at Boston University Medical Center, Lexington, MA, and Department of Epidemiology, Glaxo Wellcome, Research Triangle Park, NC. Subjects receiving two or more anticonvulsants concurrently were identified from the General Practice Research Database in the UK and their clinical records were reviewed. Of 4150 patients with refractory epilepsy, 612 (15\%) were under 20 years of age. For all subjects, including both idiopathic and acquired epilepsies, the incidence rate of highly probable SUD was $1.5 / 1000$ person-years; for highly probable and possible categories combined, the incidence rate was 2.2/1000 person-years. The respective rates for idiopathic and acquired epilepsy separately were 2.4 and 1.0/1000 person-years. Of a total of 15 subjects with SUD and epilepsy, 8 male and 7 female, 2 (13\%) were younger than 20 years. (Derby LE, Tennis P, Jick H. Sudden unexplained death among subjects with refractory epilepsy. Epilepsia Oct 1996;37:931-935). (Reprints: Dr LE Derby, Boston Collaborative Drug Surveillance Program, 11 Muzzey Street, Lexington, MA 02173).

COMMENT. Idiopathic refractory epilepsy was associated with an estimated risk of sudden unexplained death of 2.4/1000 person-years, whereas the risk with acquired refractory epilepsy was $1.0 / 1000$. A previous study has estimated a higher risk of SUD in patients with acquired epilepsy. The authors concede that relevant information on the cause of epilepsy may have been omitted in some records, leading to an overascertainment of idiopathic cases.

Male sex, the need for multiple AEDs, and use of psychotropic drugs were risk factors for SUD in a Canadian study which showed an overall rate of 
1.35/1000 person-years. (see Ped Neur Briefs Jan 1995;9:1 for review and commentary). Cardiac causes for idiopathic seizures and a normal EEG, a possible explanation for some cases of SUD, especially in adolescent males, should always be considered when AEDs are ineffective.

\section{INFANTILE SEIZURES}

\section{INFANTILE SPASMS IN DOWN SYNDROME}

The clinical characteristics, EEG abnormalities, response to therapy, and outcome of 14 patients with infantile spasms and Down syndrome were studied at the Hopital Saint Vincent de Paul, Paris (9 cases); Universita Degli Studi de Pisa, Italy (2 cases); and Hopital de La Timone, Marseille, France (3 cases). None had antecedent cardiopathy or perinatal hypoxia. Spasms began between 4 and 18 months (mean 8 months), development was delayed before seizure onset, and visual contact deteriorated after seizure onset. Interictal EEGs showed typical hypsarrhythmia with no focal abnormality. Hydrocortisone (15 $\mathrm{mg} / \mathrm{kg} /$ day for 2 weeks, and discontinuation over 2 weeks) in 10 , and vigabatrin, valproate, or pyridoxine in 4 patients, controlled spasms and hypsarrhythmia within 6 months. Five with relapses within 2 months responded to further treatments. Seven remained seizure-free, and 7 developed other types of seizures resembling idiopathic generalized epilepsies, including myoclonic jerks, absences, or generalized atonic or tonic-clonic seizures, most responding readily to a combination of valproate, ethosuximide, and diazepam. None developed Lennox-Gastaut syndrome or other chronic refractory seizure disorder. Autistic features persisted in 2. (Silva ML, Cieuta C, Guerrini R, Plouin P, Livet MO, Dulac O. Early clinical and EEG features of infantile spasms in Down syndrome. Epilepsia Oct 1996;37:977-982). (Reprints: Dr O Dulac, Hopital Saint Vincent de Paul, 82 Ave Denfert Rochereau, 75674 Paris Cedex 14, France).

COMMENT. Infantile spasms in Down syndrome have the ictal and interictal EEG characteristics of idiopathic West syndrome, they respond relatively well to therapy, and do not generally evolve into Lennox-Gastaut or other chronic epilepsy syndrome. A delay in diagnosis may contribute to a worsening of cognitive dysfunction, and parents of Down syndrome children should be alerted to the possible development of spasms in the first year.

A case of West syndrome as the initial manifestation of congenital unilateral perisylvian cortical dysplasia is reported from University Children's Hospital, Badajoz; and Galicia General University Hospital, Santiago de Compostela, Spain (Vaquerizo-Madrid J, Eiris-Punal J, Gomez-Martin H et al. Acta Neuropediatr 1996;2:132-138). The child had left hemiatrophy and paresis and was developmentally delayed. Later, he had refractory epilepsy, with complex partial, atypical absence, and atonic seizures. The interictal EEG during sleep showed right sided epileptogenic activity with contralateral spread.

\section{PYRIDOXINE-DEPENDENT SEIZURES AND MRI ABNORMALITY}

An infant with pyridoxine-dependent seizures and MRI, PET, and EEG evidence of diffuse structural or functional brain disease is reported from the University of New Mexico Health Sciences Center, Albuquerque, NM, and the UCLA School of Medicine, Los Angeles, CA. Seizures began at 10 weeks, and status epilepticus occurred four times between 3 and 7 months of age. Trials of AEDs and ACTH were partially effective, but he became encephalopathic and 